\title{
Quantitative attenuation analysis for identification of early Barrett's neoplasia in volumetric laser endomicroscopy
}

\author{
Anne-Fre Swager \\ Dirk J. Faber \\ Daniel M. de Bruin \\ Bas L. Weusten \\ Sybren L. Meijer \\ Jacques J. Bergman \\ Wouter L. Curvers \\ Ton G. van Leeuwen
}




\title{
Quantitative attenuation analysis for identification of early Barrett's neoplasia in volumetric laser endomicroscopy
}

\author{
Anne-Fre Swager, ${ }^{a}$ Dirk J. Faber, ${ }^{\text {b,* }}$ Daniel M. de Bruin, ${ }^{b}$ Bas L. Weusten, ${ }^{\text {a }}$ Sybren L. Meijer, ${ }^{c}$ \\ Jacques J. Bergman, ${ }^{a}$ Wouter L. Curvers, ${ }^{d}$ and Ton G. van Leeuwen ${ }^{b}$ \\ aUniversity of Amsterdam, Department of Gastroenterology and Hepatology, Academic Medical Center, Amsterdam, The Netherlands \\ bUniversity of Amsterdam, Department of Biomedical Engineering and Physics, Academic Medical Center, Amsterdam, The Netherlands \\ 'University of Amsterdam, Department of Pathology, Academic Medical Center, Amsterdam, The Netherlands \\ ${ }^{\mathrm{d}}$ Catharina Hospital, Department of Gastroenterology and Hepatology, Eindhoven, The Netherlands
}

\begin{abstract}
Early neoplasia in Barrett's esophagus (BE) is difficult to detect. Volumetric laser endomicroscopy (VLE) incorporates optical coherence tomography, providing a circumferential scan of the esophageal wall layers. The attenuation coefficient $\left(\mu_{\mathrm{VLE}}\right)$ quantifies decay of detected backscattered light versus depth, and could potentially improve BE neoplasia detection. The aim is to investigate feasibility of $\mu_{\mathrm{VLE}}$ for identification of early BE neoplasia. In vivo and ex vivo VLE scans with histological correlation from BE patients \pm neoplasia were used. Quantification by $\mu_{\mathrm{VLE}}$ was performed manually on areas of interest (Aols) to differentiate neoplasia from nondysplastic (ND)BE. From ex vivo VLE scans from 16 patients (13 with neoplasia), 68 Aols were analyzed. Median $\mu_{\mathrm{VLE}}$ values $\left(\mathrm{mm}^{-1}\right.$ ) were 3.7 [2.1 to 4.4 interquartile range (IQR)] for NDBE and 4.0 (2.5 to 4.9 IQR) for neoplasia, not statistically different $(p=0.82)$. Fourteen in vivo scans were used: nine from neoplastic and five from NDBE patients. Median $\mu_{\mathrm{VLE}}$ values were 1.8 (1.5 to 2.6 IQR) for NDBE and 2.1 (1.9 to 2.6 IQR) for neoplasia, with no statistically significant difference $(p=0.37)$. In conclusion, there was no significant difference in $\mu_{\mathrm{VLE}}$ values in VLE scans from early neoplasia versus NDBE. Future studies with a larger sample size should explore other quantitative methods for detection of neoplasia during BE surveillance. @ 2017 Society of Photo-Optical Instrumentation Engineers (SPIE) [DOI: 10.1117/1.JBO.22.8.086001]
\end{abstract}

Keywords: volumetric laser endomicroscopy; attenuation coefficient; Barrett's esophagus; early neoplasia.

Paper 170060RR received Feb. 13, 2017; accepted for publication Jul. 17, 2017; published online Aug. 4, 2017.

\section{Introduction}

Barrett's esophagus (BE) is a known precursor lesion for esophageal adenocarcinoma (EAC), of which the incidence has been rising steeply over the past decades. ${ }^{1,2}$ Early neoplastic lesions [like high-grade dysplasia (HGD) and/or early EAC] are difficult to distinguish within Barrett's mucosa due to their subtle appearance. Therefore, these lesions are often missed during endoscopy. The current surveillance protocol, consisting of regular white-light endoscopy and random biopsies, is therefore suboptimal, since random biopsies are prone to sampling error. $^{3,4}$

Improvement of the efficacy of Barrett's surveillance may be reached by using advanced imaging techniques. Optical coherence tomography (OCT) is a technique that measures backscattering light from different layers of tissue, creating threedimensional cross-sectional images. OCT has been shown to differentiate between normal squamous mucosa, Barrett's, and gastric mucosa. ${ }^{5-8}$ Other studies examined the ability of OCT to detect BE neoplasia. ${ }^{9-11}$ Volumetric laser endomicroscopy (VLE) is a balloon-based system incorporating optical frequency domain imaging (OFDI), enabling high-resolution, high-speed acquisition of larger tissue volumes. ${ }^{12}$ VLE is capable of scanning the esophagus circumferentially, through an inflated balloon, over a length of $6 \mathrm{~cm}$ with a lateral

*Address all correspondence to: Dirk J. Faber, E-mail: d.j.faber@amc.uva.nl resolution of $40 \mu \mathrm{m}$ (full-width half maximum, specified at the focus). ${ }^{13}$ Imaging depth in tissue is limited to about $2 \mathrm{~mm}$ and depends on tissue optical properties, and depth resolution is $7 \mu \mathrm{m}$ (as specified by the manufacturer). The lateral resolution will be decreased with distance from the focus. Within $90 \mathrm{~s}$, a scan of the superficial esophageal wall layers is performed. VLE therefore has potential to provide improved visualization of early BE neoplasia compared to current techniques. A VLE scan comprises a large amount of data (1200 circumferential frames) that need to be scrutinized for detection of subtle morphological differences of early neoplasia. Visual inspection of large sets of VLE images is complex, time consuming, and might be prone to inter- and intraobserver variation. Therefore, VLE interpretation may be a challenge for endoscopists during real-time use in the endoscopy suite. An objective quantitative measurement that enables red-flagging suspicious areas within the VLE scan could be of importance for further clinical implementation of this technique. We hypothesize that the attenuation coefficient $\mu_{\mathrm{VLE}}$, which describes the decay of detected signal with depth, is dependent on cellular changes occurring during neoplasia development (e.g., increased nucleus-cytoplasm ratio, lack of organization, and layering in the tissue), which will lead to changes in scattering properties. When derived from VLE scans, $\mu_{\mathrm{VLE}}$ could potentially provide a quantitative optical diagnosis of interrogated mucosa. Several 
studies have shown promising results for the application of the attenuation coefficient to differentiate neoplasia from various normal tissues in oral epithelial dysplasia, ${ }^{14,15}$ urothelial cancer $^{16}$ and renal tumors, ${ }^{17}$ vulvar neoplasia, ${ }^{18}$ melanomas, ${ }^{19}$ and lymph node metastasis. ${ }^{20}$ In this study, we will explore manual fitting attenuation analyses. The aim was to assess whether $\mu_{\mathrm{VLE}}$ can distinguish neoplasia from nondysplastic (ND)BE in ex vivo and in vivo VLE scans.

\section{Methods}

\subsection{Patients and Endoscopic Procedure}

All patients were included as part of previous studies by our group: NDBE patients undergoing surveillance endoscopy and patients referred for treatment of early Barrett's neoplasia (HGD/EAC). ${ }^{21,22}$ In summary, after endoscopic examination of the esophagus according to standard protocol, in vivo VLE scanning was performed. When a neoplastic lesion was detected on white-light endoscopy, it was resected according to standard guidelines for endoscopic mucosal resection. In short, the tissue containing the lesion was sucked into the cap of the endoscope after which a rubber band is released creating a pseudopolyp of tissue. Subsequently, this tissue is resected below the rubber band using an endoscopic snare with electrocoagulation. This procedure can be repeated multiple times until the entire surface of the lesion is resected. In NDBE patients, a random area was selected for resection at the discretion of the endoscopist. Subsequently, all resection samples were scanned ex vivo (see ex vivo VLE data). This study was approved by the local medical ethical committee from the Academic Medical Center in Amsterdam (protocol NTR4055, registered at Ref. 23 and NCT01862666 at Ref. 24).

\subsection{Nvision VLE Imaging System}

The Nvision VLE Imaging System (NinePoint Medical Inc., Bedford, Massachusetts) incorporates OFDI utilizing a sweptsource near-infrared laser. The system consists of a disposable optical probe, a balloon inflation system, and an imaging console consisting of a swept light source (1250 to $1350 \mathrm{~nm}$ ), interferometer, detection and data acquisition computer. At the distal end of the optical probe is a polymeric, noncompliant balloon centering the optical probe for imaging. During an automatic pullback of the optical probe, a 6-cm long, circumferential scan is obtained. In this study, a precommercial and the commercially available VLE imaging system were utilized. In vivo pullbacks contain $1200 \mathrm{~B}$-scans of 4096 A-lines each, which are obtained at $50-\mathrm{kHz}$ A-line rate resulting in a total acquisition time of $98 \mathrm{~s}$. The number of samples per A-line is 1024 for the precommercial system and 2048 for the commercial system. The maximum scanning ranges in air are 7.5 and $12 \mathrm{~mm}$, respectively. In practice, the imaging depth is limited by tissue scattering to $\sim 2 \mathrm{~mm}$ from the balloon. For both systems, the axial resolution is $\sim 7 \mu \mathrm{m}$ in tissue. The full-width at half maximum of the probe beam is $40 \mu \mathrm{m}$ at the focus. The sensitivity of the precommercial system is 105 to $110 \mathrm{~dB}$ with a dynamic range of $55 \mathrm{~dB}$; sensitivity of the commercial system is 110 to $115 \mathrm{~dB}$ with a dynamic range of $55 \mathrm{~dB}$. The precommercial system utilizes a balloon probe with an inflated diameter of $25 \mathrm{~mm}$. The focus is positioned $3.6 \mathrm{~mm}$ from zero-delay, $\sim 0.5 \mathrm{~mm}$ below the balloon-tissue interface in the tissue. Depth of focus is $0.55 \mathrm{~mm}$. The commercial system uses a balloon with inflated diameter of $20 \mathrm{~mm}$, with the focus positioned at $6.6 \mathrm{~mm}$ from zero delay, $\sim 1 \mathrm{~mm}$ below the balloontissue interface with a Rayleigh length of $0.71 \mathrm{~mm}$. For more comprehensive technical details of the optical frequency domain technology that is incorporated in the VLE imaging system, we refer to previous publications. ${ }^{25-29}$

\subsection{In Vivo VLE Data and Lesion Location Analysis}

Before inclusion in the quantitative analysis study, the scans were assessed for imaging quality, focusing on a sufficiently centered scan (intraesophageal) with equal imaging depth along the entire circumference. In the in vivo scans containing endoscopically visible neoplasia (requiring endoscopic treatment), the location of the lesion on VLE was previously determined through a meticulous analysis. Briefly, a combination of orientation marks (cautery marks visible on both VLE and white-light endoscopy) and the endoscopy and histology reports were used to construct a gridded map from the VLE scan including the lesion location. The approximate range of the lesion (VLE frame numbers and endoscopic quadrant location) was recorded.

\subsection{Ex Vivo VLE Data and Selection of Areas of Interest}

All fresh endoscopic resection specimens were scanned ex vivo with VLE, using a custom-made tubular fixture to accommodate the inflated balloon. Subsequently, the specimens were histologically processed according to standard protocol, and all histology slides were evaluated by an expert Barrett's pathologist (S.M.). In the above-mentioned study by our group, high-quality VLE-histology correlations were established according to a meticulous protocol. ${ }^{21,22}$ In short, ex vivo VLE scans from endoscopic resection specimens were correlated one-to-one with submillimeter accuracy to the corresponding histology slides, using in vivo and ex vivo placed markers. Within a VLE-histology "match," areas of interest (AoIs) consisting of one mucosal type were selected in between and beside the markers (see Fig. 1). In the current study, only the VLE AoIs containing NDBE and HGD or EAC were used for analysis. Furthermore, the AoIs were assessed for sufficient imaging quality. In addition, the histological correlates had to contain an intact epithelial layer without artifacts. If the data could not be fitted adequately [e.g., negative $\mu \mathrm{VLE}$ value, fitting not possible in the correct layer (epithelium), or inconsistent attenuation graphs over five adjacent frames], the AoIs were excluded beforehand.

\section{Quantitative Analyses of VLE}

\subsection{Attenuation Coefficient}

Scattering properties of tissue can be expressed into the attenuation coefficient $\left(\mu_{\mathrm{T}}\right)$, which measures the decay of single-backscattered light versus depth. ${ }^{30}$ In order to determine the tissuespecific attenuation coefficient from the VLE signal decay, instrumental factors have to be corrected for, such as the confocal point spread function and depth dependent sensitivity. However, a possible contribution of multiple scattered light would cause a slower decay of the VLE signal compared to single scattering. This contribution is a-priori unknown and difficult to account for Ref. 31. Therefore, in this article, we will refer to the attenuation coefficient as $\mu_{\mathrm{VLE}}$. 


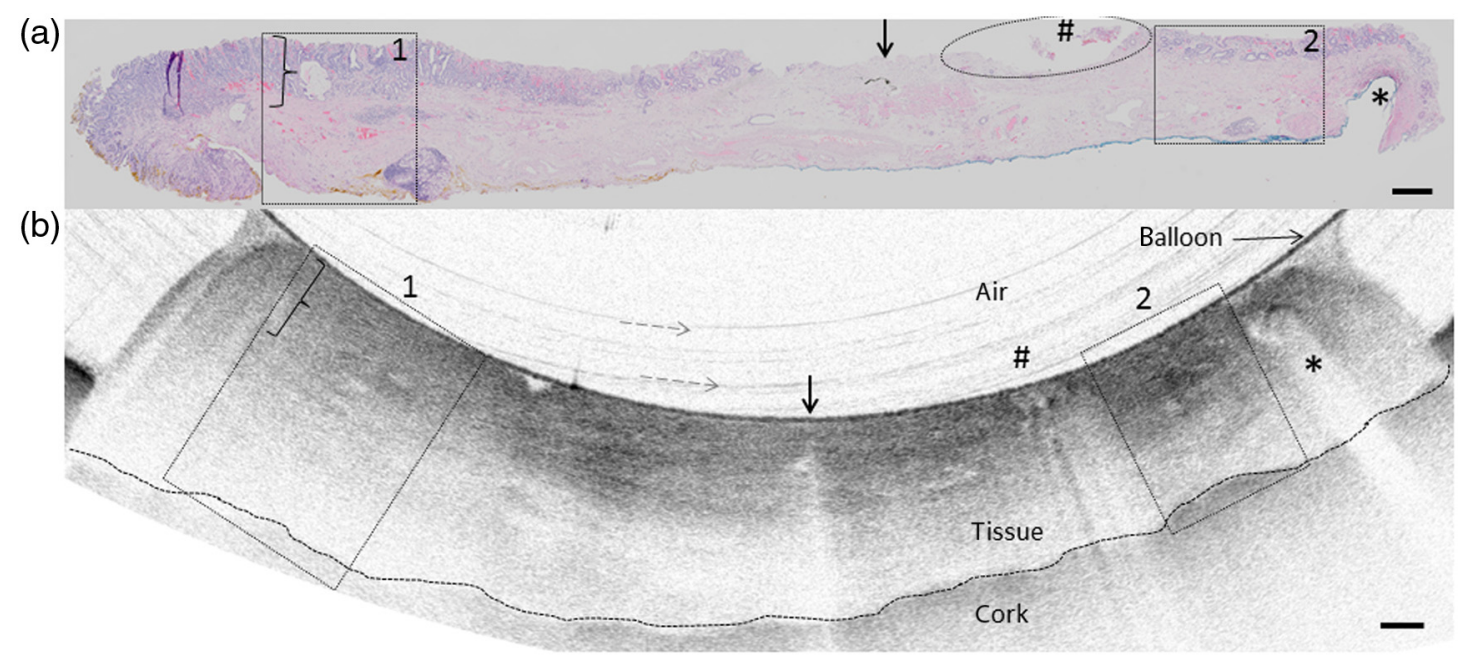

Fig. 1 (a) Example of a VLE-histology correlation of histology slide and (b) corresponding ex vivo VLE scan. Both modalities were matched one-to-one using three markers: ink (arrow), electrocoagulation (hashtag), and pin (asterisk). Area 1 contains HGD and area 2 contains NDBE. The striped gray arrows indicate imaging artifacts. Scale bars represent $500 \mu \mathrm{m}$.

\subsection{Technical Specifications}

Both systems were individually calibrated for their confocal point spread function and depth-sensitivity roll-off for subsequent corrections of the VLE signal using highly diluted Intralipid samples. In this case, the attenuation by the sample may be neglected, and the OCT signal versus depth directly measures the PSF and roll-off. For both systems individually, the focus position $z_{\mathrm{f}}$, the depth of focus $Z_{0}$, and roll-off parameter $s$ were determined. ${ }^{31}$ The ability of both systems to accurately measure attenuation was verified by measuring Intralipid samples of varying concentrations ( $0.5 \%$ to $20 \%$, undiluted). These $\mu_{\mathrm{VLE}}$ values were compared with attenuation coefficients that had been obtained previously with OCT systems operating at or near $1300 \mathrm{~nm}$ (see Fig. 2). ${ }^{32,33}$

\subsection{Manual Fitting of the Attenuation Coefficient}

Quantitative analysis was first performed on ex vivo VLE AoIs and thereafter on selected areas in in vivo VLE scans. All analyses were performed using custom-programmed plug-ins in Fiji, an open source image processing program (Ref. 34). ${ }^{35}$ First, the location of the balloon-tissue interface was determined in the unprocessed B-scans using digital edge detection. Each individual A-line was subsequently multiplied with the PSF and roll-off function of the VLE system used. Then, each A-line was shifted axially such that the balloon-tissue interface is aligned. Manual selection of the AoI was done using the match between the circumferential VLE scan and pathology slide (see Figs. 3 and 4). The average of 100 adjacent A-lines was computed, where the exact location of the $100 \mathrm{~A}$-scans within the AoI was selected at random, avoiding areas with loss of balloon-tissue contact. This results in a graph showing VLE amplitude on the vertical axis versus depth on the horizontal axis. The extent of the fit in depth was determined from the maximum depth of the epithelial layer, guided by corresponding histology. The epithelial layer (ranging from 200 to $500 \mu \mathrm{m}$ maximum depth) was used because it contains the most relevant clinical information regarding mucosal type and diagnosis. For each AoI or in vivo area, the OCT signal as a function of optical depth was fitted with an exponential function, with the signal amplitude and $\mu_{\mathrm{VLE}}$ as running parameters. For each lesion, in total, five B-scans were analyzed, two adjacent B-scans on both sides of the B-scan that was matched to histology. These five $\mu_{\mathrm{VLE}}$ values were averaged, resulting in a final $\mu_{\mathrm{VLE}}$ value. ${ }^{36}$

\subsection{Statistics}

SPSS (Statistical Package for the Social Sciences 23; SPSS Inc., Chicago, Illinois) was used to perform statistical analysis. For descriptive statistics, mean \pm standard deviation (SD) was used

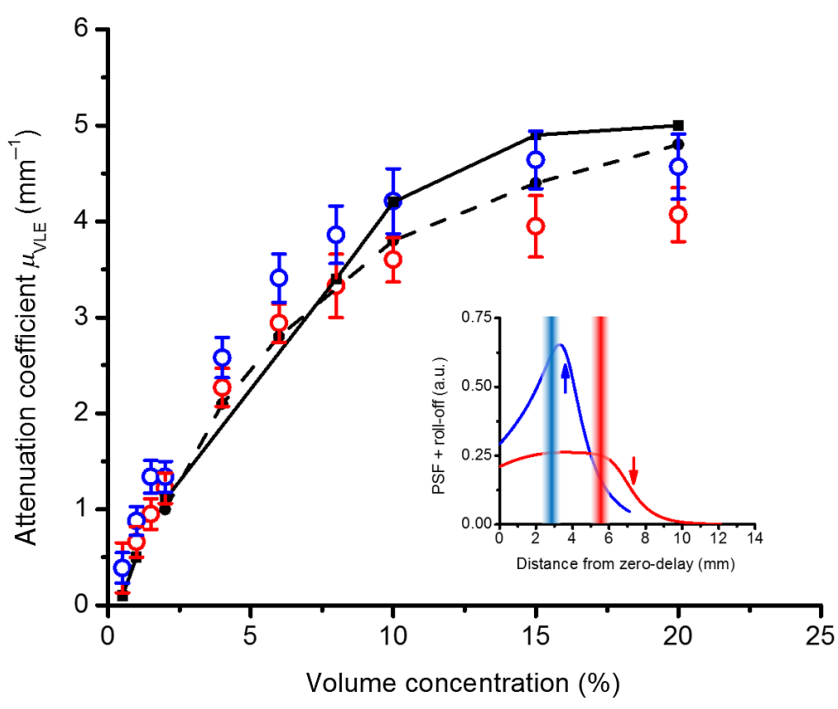

Fig. 2 Attenuation coefficients determined with the precommercial (blue circles) and commercial (red circles) VLE systems. The dashed $\left(K \mathrm{Kalkman}^{32}\right)$ and solid $\left(\mathrm{Kodach}^{33}\right)$ black lines show reference measurements from previous publications using different $1300-\mathrm{nm}$ OCT systems. The inset shows combined confocal point spread function and sensitivity roll-off for both VLE systems as function of distance from zero-delay. Arrows indicate the position of the focus in an A-line, at 3.6 and $6.6 \mathrm{~mm}$ from zero-delay, respectively. The bars show the average location of the balloon edge in both probe configurations. 
(a)

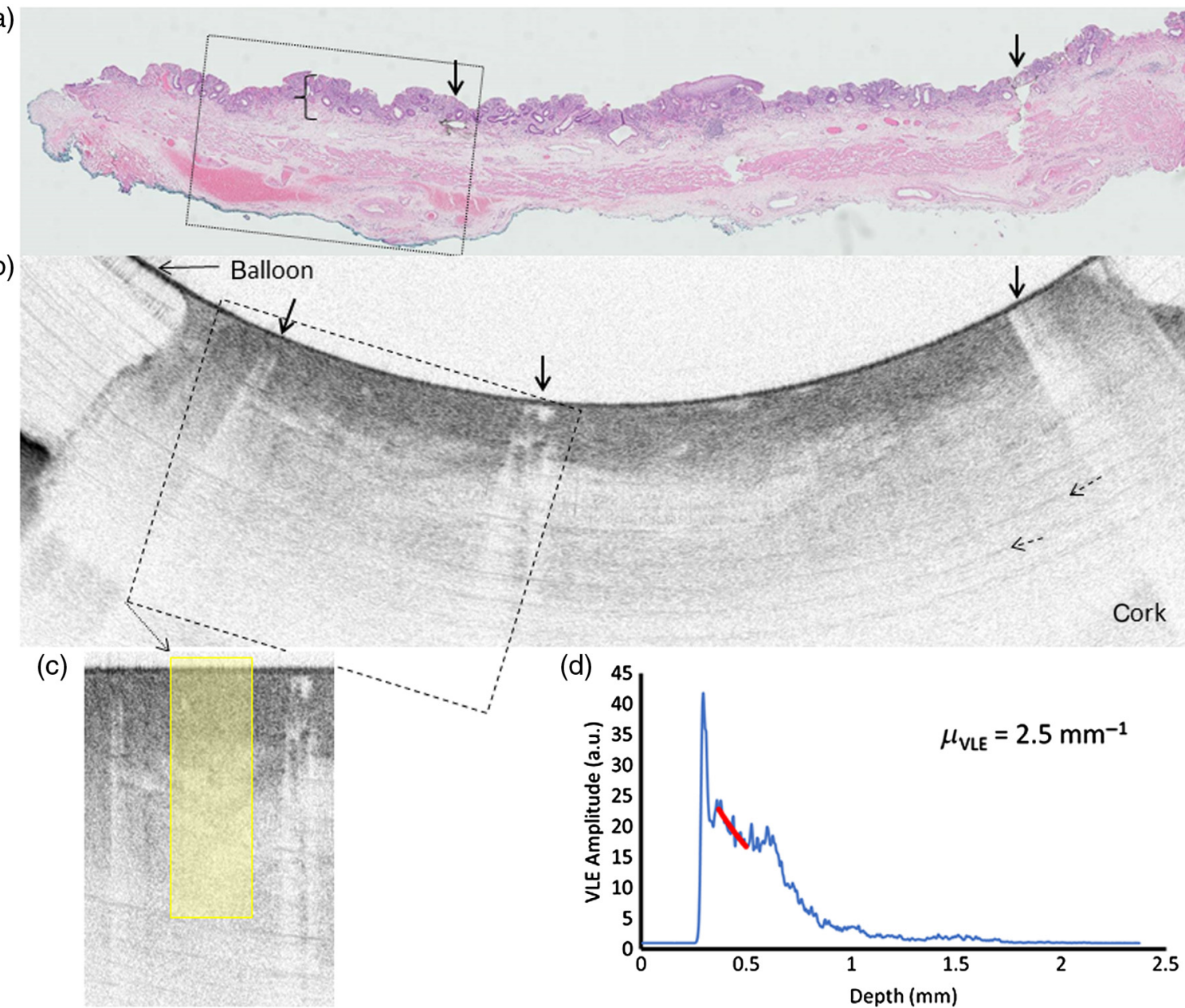

Fig. 3 Analysis on ex vivo VLE image. Area of interest (square) indicated in the histological correlate (a) and the VLE frame (b), containing NDBE. Vertical arrows indicate ink markers in (a) and (b) that were used to correlate VLE with histology. Image artifacts caused by reflection of the balloon are indicated with a striped arrow (in b). In the straightened Aol (c), the yellow square indicates the $100 \mathrm{~A}$-lines over which the fit analysis was performed (d). In (d), the attenuation coefficient graph depicts the VLE signal with gray values on the vertical axis and the depth in $\mathrm{mm}$ on the horizontal axis. The black vertical lines and red line on the graph indicate the exact area that was fitted, corresponding with the superficial epithelial layer on histology (accolade). Scale bars represent $500 \mu \mathrm{m}$.

in case of a normal distribution of variables, and median [interquartile range (IQR)] was used for variables with a skewed distribution. The Mann-Whitney U test was performed to compare the average attenuation coefficient values from VLE AoIs and in vivo scans containing neoplasia versus NDBE tissue.

\section{Results}

\subsection{Patient Demographics}

In vivo and ex vivo VLE scans from, in total, 21 patients were used; from seven patients, only ex vivo scans, from five patients, only in vivo scans and from nine patients both in vivo and ex vivo scans were used. The mean age was $68(\mathrm{SD} \pm 8)$ years and 15 patients were men. Five were NDBE patients and 16 patients were known with neoplasia. The worst histological outcome per patient was EAC in 12, HGD in 3, low-grade dysplasia in 1 , and NDBE in 5. Median circumferential extent of the BE segment was $2 \mathrm{~cm}$ (IQR 1 to $5 \mathrm{~cm}$ ), and the maximum extent was $5 \mathrm{~cm}$ (IQR 4 to $8 \mathrm{~cm}$ ).

\subsection{VLE Data}

In this study, 68 AoIs from ex vivo VLE images were quantitatively analyzed. Forty-two VLE AoIs, of which two were taken with the precommercial system, contained neoplasia (17 HGD, 25 EAC) and 26, of which 12 were taken with the precommercial system, contained NDBE on corresponding histology. Outside those 68 AoIs, 5 were excluded beforehand because the data could not be fitted adequately (e.g., negative $\mu \mathrm{VLE}$ value). In total, 14 in vivo scans were used to perform quantitative analyses: nine from patients with endoscopically visible neoplasia (all scans imaged with commercial system) and five from NDBE patients undergoing surveillance (precommercial system). From each neoplastic scan, three different areas in the neoplastic lesion and three different areas in the NDBE scan were analyzed.

\subsection{Validation Measurements}

Figure 2 shows measurements of the attenuation coefficient versus volume fraction of Intralipid $20 \%$, obtained with both 
(a)

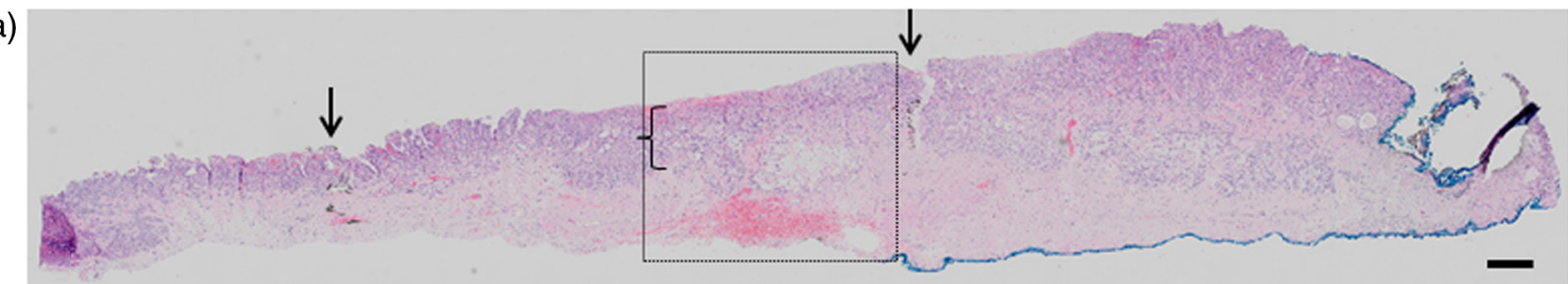

(b)

(c)

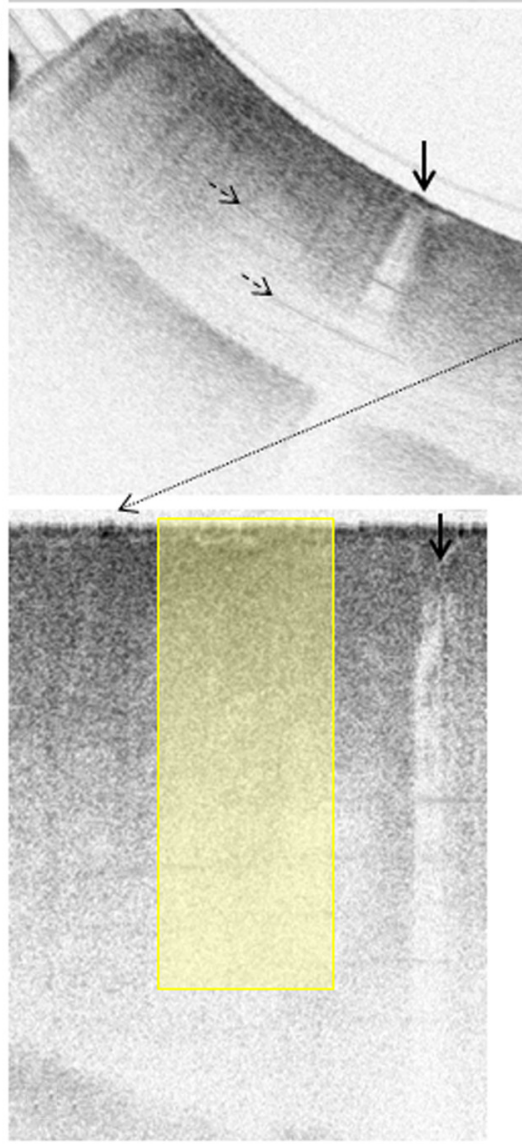

(d)

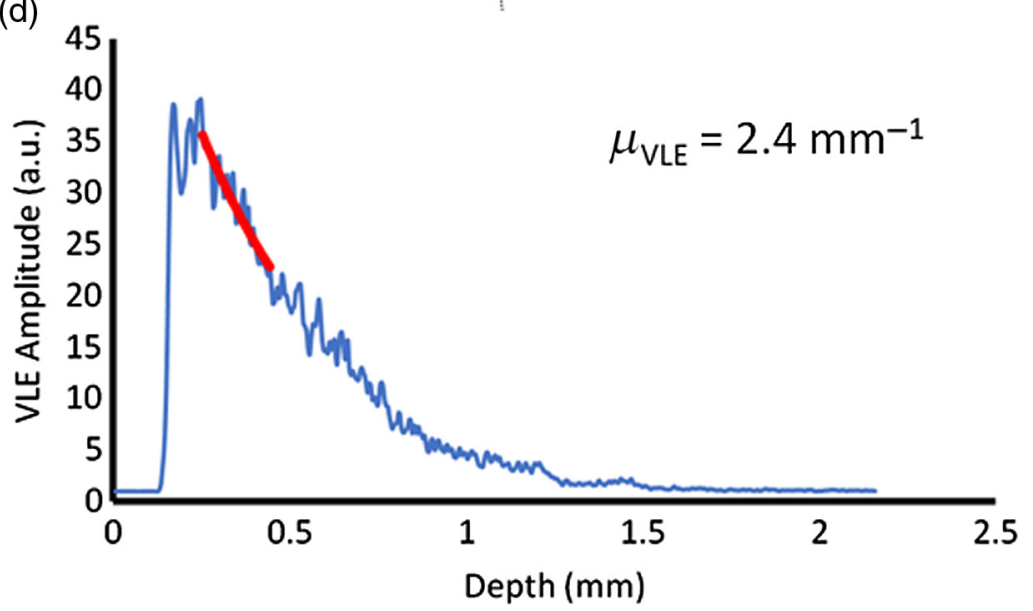

Fig. 4 Analysis on ex vivo VLE image. Area of interest (square) indicated in the histological correlate (a) and the VLE frame (b) containing EAC. Vertical arrows indicate ink markers in (a) and (b) that were used to correlate VLE with histology. Image artifacts caused by reflection of the balloon are indicated with a striped arrow (in b). In the straightened Aol (c), the yellow square indicates the $100 \mathrm{~A}$-lines over which the fit analysis was done (d). In (d), the attenuation coefficient graph depicts the VLE signal with gray values on the vertical axis and the depth in $\mathrm{mm}$ on the horizontal axis. The red line on the graph indicates the exact area that was fitted, corresponding with the superficial part of the epithelial layer on histology (accolade). Scaling: the histology slide is on average 1 to $2 \mathrm{~mm}$ thick, as is the thickness of the tissue on the corresponding VLE scan, with a width of a quarter of the circumference of the balloon $(\approx 16 \mathrm{~mm})$. Scale bars represent $500 \mu \mathrm{m}$.

the precommercial and commercial VLE systems. Additionally, reference measurements are shown that were obtained previously with different laboratory OCT systems operating at center wavelengths near $1300 \mathrm{~nm}$. The confocal point spread function and sensitivity roll-off versus distance from zero-delay for both VLE systems is also shown. Good mutual correspondence between attenuation coefficients is obtained for Intralipid volume fractions up to $10 \%$, demonstrating correct inclusion of the point spread function and roll-off in the data analysis.

\subsection{Quantitative Analyses}

Figures 3 and 4 depict the manual analysis on ex vivo VLE images, showing a VLE-histology match and the area of interest with the attenuation coefficient graph. Median $\mu_{\mathrm{VLE}}$ values $\left(\mathrm{mm}^{-1}\right)$ of the different mucosa types were 3.7 (2.1 to 4.4 IQR) for NDBE and 4.0 (2.5 to $4.9 \mathrm{IQR}$ ) for neoplasia [see Fig. 5(a)]. There was no statistically significant difference between the two groups $(p=0.82)$. Figure 6 depicts the manual analysis on in vivo scans. Median $\mu_{\mathrm{VLE}}$ values $\left(\mathrm{mm}^{-1}\right)$ of the different mucosa types were 1.8 (1.5 to $2.6 \mathrm{IQR})$ for NDBE and 2.1 (1.9 to $2.6 \mathrm{IQR}$ ) for neoplasia [see Fig. 5(b)]. There was no statistically significant difference between the two groups $(p=0.37)$.

\section{Discussion}

The current Barrett's surveillance protocol is not optimal because early neoplastic lesions are often missed during 
(a)

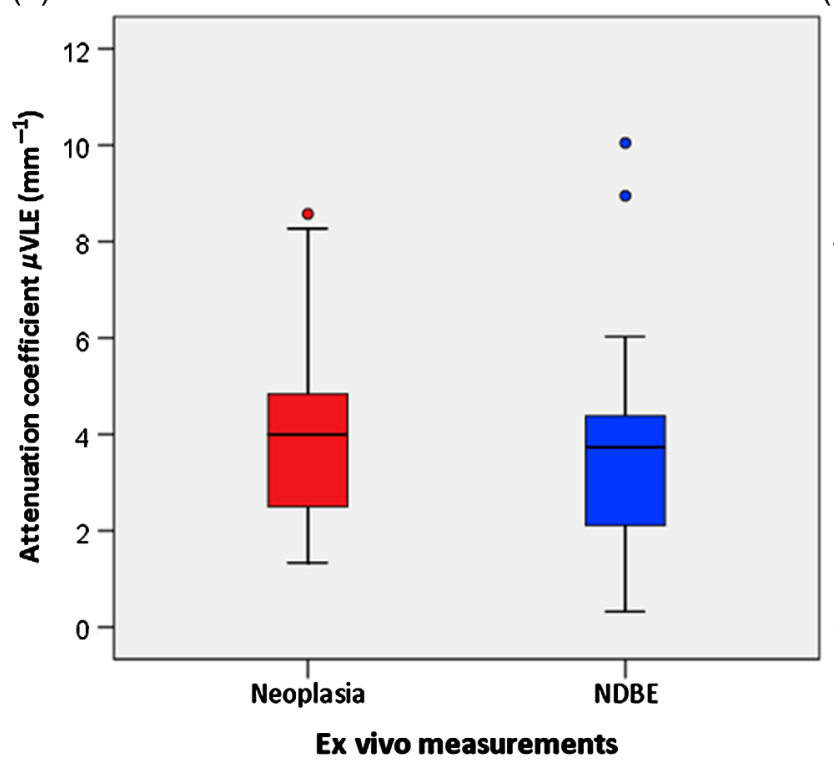

(b)

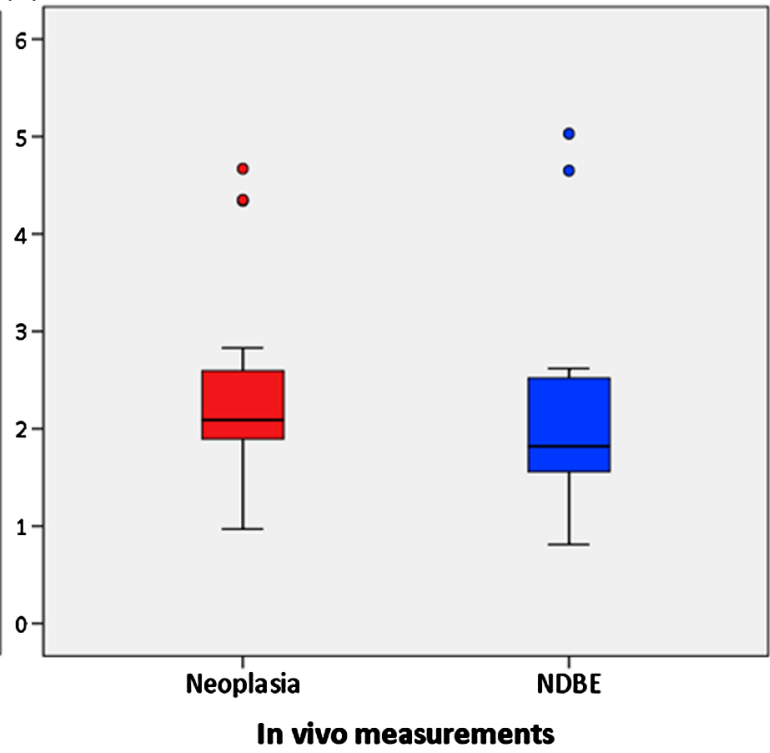

Fig. 5 (a) Box plot of attenuation coefficients of NDBE and neoplasia (HGD/EAC) of ex vivo Aols. (b) Box plot of attenuation coefficients of NDBE and neoplasia (HGD/EAC) areas of in vivo scans. Horizontal lines represent median values, boxes indicate IQR, and error bars indicate range.

(a)

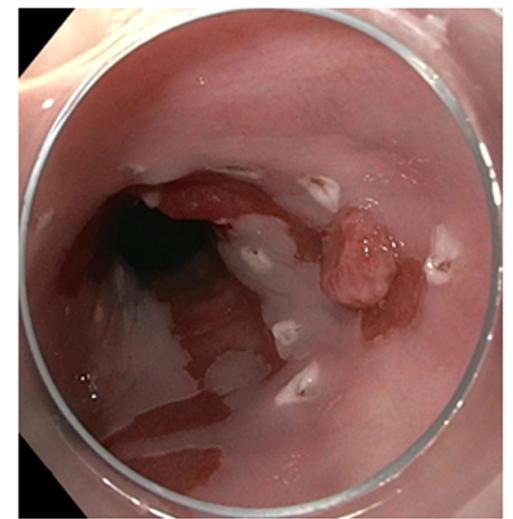

(b)

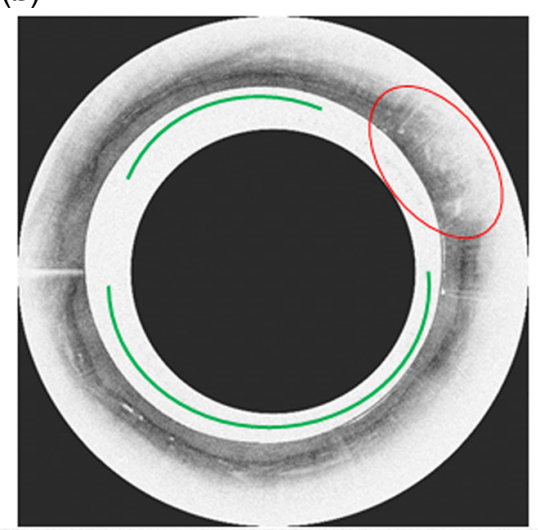

(c)

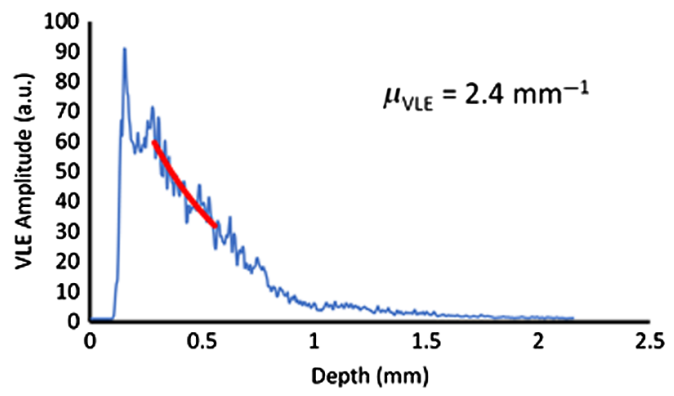

(d)

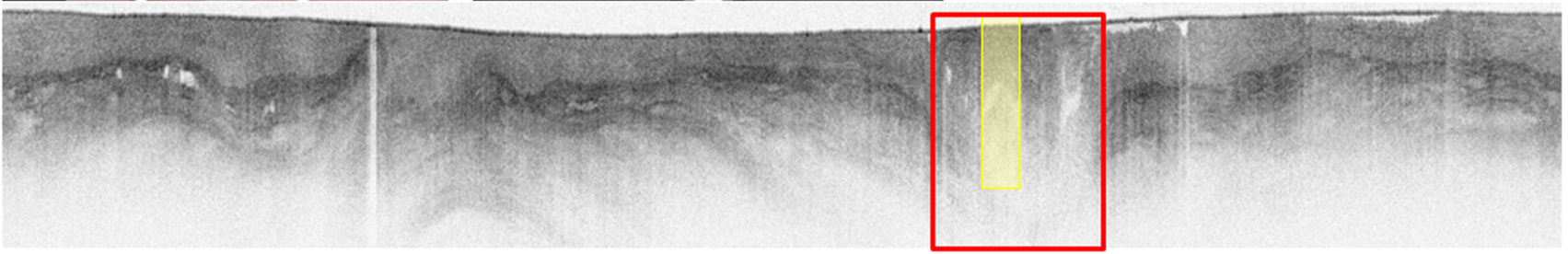

Fig. 6 (a) Analysis on in vivo VLE scan. The white-light endoscopy image shows the nodular neoplastic lesion in the esophagus, which is delineated by cautery marks (white spots). In the VLE scan, the location of the lesion was determined through meticulous analysis. (b) On the circular view VLE image, the lesion (red oval) is visible by lack of layering and irregular glandular structures, surrounded by mostly squamous epithelium (green lines), characterized by the layered pattern. On the straight VLE image, (c) a quantitative fitting analysis was performed in the (d) neoplastic area (red square). The yellow box indicates 100 A-lines over which the fit was performed on the graph (c). The graph represents the decay of backscattered light in gray values with depth in $\mathrm{mm}$.

endoscopy due to their subtle appearance. The advanced imaging system VLE has the potential to improve detection of early Barrett's neoplasia by providing a quick circumferential scan of the entire BE, also visualizing the subsurface layers. However, one VLE scan comprises 1200 frames consisting of subtle gray-shade architectures that need to be scrutinized by the endoscopist during the procedure. Therefore, interpretation of VLE scans is complex and prone to inter- and intraobserver variation. Hence, there is a necessity for an objective, quantitative assessment method of VLE data to aid the endoscopist. To 
our knowledge, this is the first study examining the attenuation coefficient $\mu_{\mathrm{T}}$ in VLE images to distinguish neoplasia from NDBE tissue. Hence, this study was developed according to stage $2 \mathrm{a}$ from the IDEAL model. ${ }^{37}$ The IDEAL framework, which is designed for evaluation of complex interventions like invasive diagnostics and therapies, defines the following stages of research: 1 -innovation/idea, $2 \mathrm{a}$-development, $2 \mathrm{~b}$ -exploration, 3-assessment, and 4-long-term follow-up. ${ }^{37}$ The present study corresponds to stage 2a. The first step of this study was to perform $\mu_{\mathrm{VLE}}$ analyses unblinded, with the corresponding histology guiding the fitting analysis (epithelial layer thickness). The particular strength of this study was the exact VLE-histology matching. Accordingly, the fitting analysis was performed at the correct depth in the tissue by determining the maximal thickness of the epithelial layer using the corresponding histology. The epithelium is used by the pathologist to diagnose the tissue and therefore, we hypothesized that this layer contains the most significant information for fitting.

The $\mu_{\mathrm{VLE}}$ quantification method is hypothesized to detect morphological and biochemical changes occurring during neoplasia development. Quantitative $\mu_{\mathrm{OCT}}$ analysis was reported to differentiate between benign and (pre)malignant tissue in several other organs. ${ }^{14,16-18,38-41}$ Volumetric OCT imaging data of brain cancer tissue had significantly lower optical attenuation values at both cancer core and infiltrated zones when compared with noncancer white matter. ${ }^{40}$ A subsequent study by the same group developed a new attenuation mapping algorithm using OCT intensity images from freshly resected human brain cancer, which was capable of accurate quantitative interpretation of OCT images in real-time during brain surgery. ${ }^{41}$ In oral tissue biopsies, $\mu_{\mathrm{OCT}}$ was shown to differentiate between normal tissue and oral epithelial dysplasia. ${ }^{14}$ Furthermore, that study demonstrated concise color maps constructed from scattering gradients, which could potentially present pathological information to the clinician. The difference in $\mu_{\mathrm{OCT}}$ or $\mu_{\mathrm{VLE}}$ values is most likely explained by cellular changes occurring during neoplasia development, which lead to changes in scattering properties. The exact mechanism is, however, still unclear and here, we will discuss several theories. The morphological changes that occur in tissue will change light scattering, depending on the size, refractive index, and spatial distribution of all particles. One theory is that in neoplastic tissue, the larger cell nuclei lead to a higher nucleus/cytoplasm ratio, causing more scattering and a higher VLE signal. Consequently, in neoplasia, the $\mu_{\mathrm{VLE}}$ value would be higher, because the decay of detected backscattered light increases more compared to NDBE tissue with smaller nuclei. However, there is more to the cell than the nucleus. In a study examining changes in scattering in apoptosis, it was found that these changes could potentially be caused by oxidative stress-induced mitochondrial swelling, causing an increase in scattering. ${ }^{42}$ On the other hand, a previous study on melanomas showed a significantly lower value for neoplastic lesions compared to benign lesions. ${ }^{38}$ Potential explanations mentioned in this study were the differences in architecture and mitosis activity in the cells. Further studies on in vivo VLE data will be necessary to unravel the underlying mechanisms.

The basis for this study was a previous pilot study with a small ex vivo VLE dataset (of which the images were incorporated in this study) showing a significant difference in the median $\mu_{\mathrm{VLE}}$ values between the neoplastic and NDBE VLE images. ${ }^{43}$ Disappointingly, though, our current results with both ex vivo and in vivo data show no difference in $\mu_{\mathrm{VLE}}$ values. Ex vivo VLE data from endoscopic resection specimens differ from in vivo data. Deceased tissue, although freshly imaged, inevitably results in different VLE appearances of certain structures than living tissue; e.g., structures like glands or blood vessels may have collapsed. Furthermore, the custom-made tubular fixture in which the ex vivo specimens were scanned caused slight pressure on the tissue, creating a different situation from in vivo scanned tissue. ${ }^{21}$ Nevertheless, for the ex vivo VLE images, both the histology and VLE images were carefully examined on artifacts caused by resection, and otherwise excluded from analysis. The next step after analysing ex vivo images was to perform analyses on in vivo data, since in vivo VLE is more relevant in clinical practice. Strikingly, the median values from the ex vivo data were about twice as high as the median values from the in vivo data. Apart from abovementioned differences between ex vivo and in vivo VLE data, we could not explain this difference in median values. Our data were taken partly with the commercial and precommercial systems. Group sizes were too small to allow separate subgroup analysis of both systems. Paramount to this study is proper calibration of the confocal point spread function and roll-off, to allow comparison between measurements obtained with both systems. Through Intralipid measurements, it was verified that reliable attenuation coefficient analysis could be performed in data from both the precommercial and commercial systems. For low volume fractions, single backscattered light is expected to dominate the signal. Due to volume concentration-dependent scattering, the attenuation coefficient does not increase linearly with volume concentration. ${ }^{31}$ At the highest volume fraction, multiple scattering may also contribute to the signal. The extent of this effect depends on the confocal properties of the systems and can therefore be different for the precommercial and commercial systems. Since this is the first study using the attenuation coefficient to analyze VLE data, we designed a manual fitting protocol. During this pioneering process, we encountered some challenges, which will be discussed here. First, manual fitting of the attenuation coefficient was complex because of several reasons: Although in the majority of the graphs decay could be measured, sometimes the shape of the graph did not allow for fitting a decay, even after averaging over 100 Alines in five adjacent B-scans. In some cases, this resulted in fitting over a smaller area or exclusion of the fit if the value was negative. There is no certain explanation for the cause of the negative values. In theory, the VLE balloon could cause compression of the tissue layers, which could cause high scattering. However, since all resection specimens were imaged in a similar fashion using the same pressure on tissue, the majority of the AoIs would then be expected to have a negative attenuation value. Although the fitting depth was defined through careful analysis of the epithelial thickness in the corresponding histology slide, determination of the exact boundaries of the fit was complex. Second, manual fitting remains partly subjective and dependent on the reviewer. On the other hand, the interobserver variability of performing fitting analyses by trained personnel has recently been studied and showed to be negligible. ${ }^{44}$ According to that study, determination of $\mu_{\mathrm{VLE}}$ does not require extensive training. The small variation in values per observer can be accepted. Still, automated analysis of the attenuation coefficient may circumvent the influence of the reviewer. Presenting the results, e.g., by means of a color-map as an overlay on $e x$ vivo and in vivo VLE images could be helpful by red-flagging 
suspicious areas during endoscopy. A Mann-Whitney U test was used to confirm that there was no statistically significant difference regarding the $\mu_{\mathrm{VLE}}$ values between the NDBE and neoplastic groups for both in vivo and ex vivo data (as was expected by visual comparison of the boxplots in Fig. 5). This test does not take into account paired data. The ex vivo VLE data were paired on multiple levels, since some AoIs originated from the same patient, the same endoscopic resection specimen or even from the same histology slide. From the in vivo scans, three areas per scan were used. However, the current datasets were too small to use a mixed-effects' model in SPSS, which could correct for any correlations between VLE measurements in the same patient. Quantitative analyses that could function as a real-time red-flagging tool for suspicious areas in VLE scans will have great potential for the future clinical application of VLE and other advanced imaging techniques in endoscopy. Other quantitative techniques in the field of image analysis have been reported for classification of dysplasia. ${ }^{45-47} \mathrm{Qi}$ et al. ${ }^{47}$ also utilized tissue backscattering and the attenuation coefficient to represent the intensity characteristic of OCT images. Furthermore, a clinically inspired computer algorithm that performs image analysis to detect BE neoplasia on VLE shows to have a very promising performance. ${ }^{45,48}$ In conclusion, in this study, there was no significant difference in $\mu_{\mathrm{VLE}}$ values in early Barrett's neoplasia versus NDBE, in ex vivo and in vivo VLE data. Future studies using in vivo VLE data in a larger sample size should further explore other and preferably automated real-time quantitative methods, such as computer-aided detection of neoplasia.

\section{Disclosures}

Bergman receives research support from Olympus Endoscopy, Fuji-film, Boston Scientific, GI Solutions Medtronic, Erbe, Ninepoint Medical, C2 therapeutics, Cernostics, Interpace, Lumen-R. The other authors have no conflicts of interest to declare.

\section{Acknowledgments}

We want to thank Mr. Brandt for programming the custom-made plug-ins for Fiji and his assistance in preparing the VLE data. This study was supported by an unrestricted grant from NinePoint Medical Inc. (NinePoint Medical Inc., Bedford, Massachusetts).

\section{References}

1. P. N. Post, P. D. Siersema, and H. Van Dekken, "Rising incidence of clinically evident Barrett's oesophagus in The Netherlands: a nationwide registry of pathology reports," Scand. J. Gastroenterol. 42(1), 17-22 (2007).

2. H Pohl, B. Sirovich, and H. G. Welch, "Esophageal adenocarcinoma incidence: are we reaching the peak?" Cancer Epidemiol. Biomarkers Prev. 19(6), 1468-1470 (2010).

3. E. R. Tschanz, "Do $40 \%$ of patients resected for Barrett esophagus with high-grade dysplasia have unsuspected adenocarcinoma?" Arch. Pathol. Lab. Med. 129(2), 177-180 (2005).

4. L. G. Gordon et al., "Cost-effectiveness of endoscopic surveillance of non-dysplastic Barrett's esophagus," Gastrointest. Endoscopy 79(2), 242-256 (2014).

5. M. J. Cobb et al., "Imaging of subsquamous Barrett's epithelium with ultrahigh-resolution optical coherence tomography: a histologic correlation study," Gastrointest. Endoscopy 71(2), 223-230 (2010).

6. M. V. Sivak et al., "High-resolution endoscopic imaging of the GI tract using optical coherence tomography," Gastrointest. Endoscopy 51(4), 474-479 (2000).
7. J. A. Evans et al., "Identifying intestinal metaplasia at the squamocolumnar junction by using optical coherence tomography," Gastrointest. Endoscopy 65(1), 50-56 (2007).

8. J. M. Poneros et al., "Diagnosis of specialized intestinal metaplasia by optical coherence tomography," Gastroenterology 120(1), 7-12 (2001).

9. J. A. Evans et al., "Optical coherence tomography to identify intramucosal carcinoma and high-grade dysplasia in Barrett's esophagus," Clin. Gastroenterol. Hepatol. 4(1), 38-43 (2006).

10. G. Isenberg et al., "Accuracy of endoscopic optical coherence tomography in the detection of dysplasia in Barrett's esophagus: a prospective, double-blinded study," Gastrointest. Endoscopy 62(6), 825-831 (2005).

11. S. Jäckle et al., "In vivo endoscopic optical coherence tomography of esophagitis, Barrett's esophagus, and adenocarcinoma of the esophagus," Endoscopy 32(10), 750-755 (2000).

12. B. E. Bouma et al., "Fourier-domain optical coherence tomography: recent advances toward clinical utility," Curr. Opin. Biotechnol. 20(1), 111-118 (2009).

13. H. C. Wolfsen et al., "Safety and feasibility of volumetric laser endomicroscopy in patients with Barrett's esophagus (with videos)," Gastrointest. Endoscopy 82(4), 631-640 (2015).

14. P. H. Tomlins et al., "Scattering attenuation microscopy of oral epithelial dysplasia," J. Biomed. Opt. 15(6), 066003 (2010).

15. O. K. Adegun et al., "Quantitative analysis of optical coherence tomography and histopathology images of normal and dysplastic oral mucosal tissues," Lasers Med. Sci. 27(4), 795-804 (2012).

16. M. T. Jenneke Bus et al., "Volumetric in vivo visualization of upper urinary tract tumors using optical coherence tomography: a pilot study," J. Urol. 190(6), 2236-2242 (2013).

17. K. Barwari et al., "Differentiation between normal renal tissue and renal tumours using functional optical coherence tomography: a phase I in vivo human study," BJU Int. 110(8 Pt B), E415-E420 (2012).

18. R. Wessels et al., "Optical coherence tomography in vulvar intraepithelial neoplasia," J. Biomed. Opt. 17(11), 116022 (2012).

19. R. Wessels et al., "Functional optical coherence tomography of pigmented lesions," J. Eur. Acad. Dermatol. Venereol. 29(4), 738-744 (2015).

20. L. Scolaro et al., "Parametric imaging of the local attenuation coefficient in human axillary lymph nodes assessed using optical coherence tomography," Biomed. Opt. Express 3(2), 366-379 (2012).

21. A. Swager et al., "Volumetric laser endomicroscopy in Barrett's esophagus: a feasibility study on histological correlation," Dis. Esophagus 29, 505-512 (2016).

22. A. Swager et al., "Identification of volumetric laser endomicroscopy features predictive for early neoplasia in Barrett's esophagus using high-quality histological correlation," Gastrointest. Endoscopy 85(5), 918-926 (2017).

23. Nederlands Trial Register, www.trialregister.nl.

24. www.clinicaltrials.gov.

25. S. H. Yun et al., "Comprehensive volumetric optical microscopy in vivo," Nat. Med. 12, 1429-1433 (2006).

26. S Yun et al., "High-speed optical frequency-domain imaging," Opt. Express 11(22), 2953-2963 (2003).

27. B. J. Vakoc et al., "Comprehensive esophageal microscopy by using optical frequency-domain imaging (with video)," Gastrointest. Endoscopy 65(6), 898-905 (2007).

28. M. J. Suter et al., "Comprehensive microscopy of the esophagus in human patients with optical frequency domain imaging. Array, editor," Gastrointest. Endoscopy 68(4), 745-753 (2008).

29. M. J. Suter et al., "Esophageal-guided biopsy with volumetric laser endomicroscopy and laser cautery marking: a pilot clinical study," Gastrointest. Endoscopy 79(6), 886-896 (2014).

30. D. Faber et al., "Quantitative measurement of attenuation coefficients of weakly scattering media using optical coherence tomography," Opt. Express 12(19), 4353-4365 (2004).

31. M. Almasian et al., "Validation of quantitative attenuation and backscattering coefficient measurements by optical coherence tomography in the concentration-dependent and multiple scattering regime," J. Biomed. Opt. 20(12), 121314 (2015).

32. J. Kalkman et al., "Multiple and dependent scattering effects in Doppler optical coherence tomography," Opt. Express 18(4), 38833892 (2010). 
33. V. M. Kodach et al., "Quantitative comparison of the OCT imaging depth at $1300 \mathrm{~nm}$ and $1600 \mathrm{~nm}, "$ Sci. Biomed. Opt. Express 1(1), 176-185 (1991)

34. Fiji is just ImageJ, http://fiji.sc/Fiji.

35. J. Schindelin et al., "Fiji-an open source platform for biological image analysis," Nat. Methods 9(7), 676-682 (2012).

36. F. J. van der Meer et al., "Quantitative optical coherence tomography of arterial wall components," Lasers Med. Sci. 20(1), 45-51 (2005).

37. P. McCulloch et al., "No surgical innovation without evaluation: the IDEAL recommendations," Lancet 374(9695), 1105-1112 (2009).

38. R. Wessels et al., "Functional optical coherence tomography of pigmented lesions," J. Eur. Acad. Dermatol. Venereol. 29(4), 738-744 (2015).

39. L. Scolaro et al., "Parametric imaging of the local attenuation coefficient in human axillary lymph nodes assessed using optical coherence tomography," Biomed. Opt. Express 3(2), 366-379 (2012).

40. C. Kut et al., "Detection of human brain cancer infiltration ex vivo and in vivo using quantitative optical coherence tomography," Sci. Transl. Med. 7(292), 292ra100 (2015).

41. W. Yuan et al., "Robust and fast characterization of OCT-based optical attenuation using a novel frequency-domain algorithm for brain cancer detection," Sci. Rep. 7, 44909 (2017).

42. D. M. de Bruin et al., "Assessment of apoptosis induced changes in scattering using optical coherence tomography," J. Biophotonics 9(9), 913-923 (2016)
43. A. Swager et al., "Quantitative analysis of volumetric laser endomicroscopy of histologically correlated images potentially identifies early neoplasia in Barrett's esophagus," Gastroenterology 146(5), S-10 (2015).

44. R. Wessels et al., "Learning curve and interobserver variance in quantification of the optical coherence tomography attenuation coefficient," J. Biomed. Opt. 20(12), 121313 (2015).

45. S. R. Klomp et al., "Evaluation of image features and classification methods for Barrett's cancer detection using VLE imaging," Proc. SPIE 10134, 101340D (2017).

46. X. Qi et al., "Computer-aided diagnosis of dysplasia in Barrett's esophagus using endoscopic optical coherence tomography," J. Biomed. Opt. 11(4), 044010 (2006).

47. X. Qi et al., "Image analysis for classification of dysplasia in Barrett's esophagus using endoscopic optical coherence tomography," Biomed. Opt. Express 1(3), 825-847 (2010).

48. A. Swager et al., "Feasibility of a computer algorithm for detection of early Barrett's neoplasia using volumetric laser endomicroscopy," Gastroenterology 150(4), S56 (2016).

Biographies for the authors are not available. 